\title{
GOBERNANZA Y POLÍTICA LABORAL EN MÉXICO: OPCIONES ANTE LA PRECARIEDAD ${ }^{1,2}$
}

Governance and Labor Policy in Mexico:

Options against precariousness

Recibido: 21 de diciembre de 2020

Aceptado: 12 de marzo de 2021

1- Rosa Isabel Medina Parra*. Grado académico: Doctora en Ciencias Administrativas. Adscripción: El Colegio de la Frontera Norte, Departamento de Estudios de Administración Pública. Correo electrónico: imedina@colef.mx. ORCID ID: https://orcid.org/0000-0002-7864-1575. *autor de correspondencia

2- José María Ramos González. Grado académico: Doctor en Gobierno y Administración Pública. Adscripción: El Colegio de la Frontera Norte, Departamento de Estudios de Administración Pública. Correo electrónico: ramosjm@colef.mxORCID ID: https://orcid.org/0000-0001-6440-6470 
Rua

\title{
RESUMEN
}

Las principales aproximaciones a la gobernanza plantean que ésta gira en torno a la eficiente gestión de las políticas públicas. Así, el presente trabajo de investigación se orienta a analizar la incidencia de la gobernanza en la eficacia del Programa Sectorial de Trabajo y Previsión Social 2020-2024, específicamente bajo el contexto de los efectos de la pandemia del Covid-19; de entre los cuales destacan la desigualdad social y precariedad laboral, cuya agudización afectará el nivel de vida de los grupos en situación de vulnerabilidad en México. Por lo que es fundamental impulsar un modelo de gobernanza que promueva el PSTPS generando alternativas de empleo.

Palabras clave: Gobernanza, Laboral, Precariedad, Política, Covid-19.

\begin{abstract}
The main approaches to governance suggest that it circles around the efficient management of public policies. Therefore, this research work is aimed to analyze governance incidence in the Sectoral Program for Work and Social Welfare 2020-2024 effectiveness, specifically in the context of Covid-19 pandemic effects; highlighting among are social inequality and occupational precariousness, which sharpening will affect the standard of living of vulnerable groups in Mexico. So, it is essential to impulse a governance model that promotes PSTPS by generating employment alternatives.
\end{abstract}

Keywords: Governance, Labor, Precariousness, Politics, Covid-19.

Clasificación JEL: M12, M48. 


\section{Introducción}

$\mathrm{L}$ a literatura académica refleja que entre las distintas aproximaciones teóricas relativas a la gobernanza, destacan aquellas que refieren que ésta gira en torno a la eficiente gestión de las políticas públicas (Zurbiggen, 2011); por tanto, el presente trabajo de investigación tiene como objetivo analizar la incidencia de la gobernanza en la eficacia del Programa Sectorial de Trabajo y Previsión Social 2020-2024, específicamente bajo el contexto de los efectos de la pandemia del Covid-19: de entre los cuales destacan la desigualdad social y precariedad laboral, cuya agudización afectará el nivel de vida de los grupos en situación de vulnerabilidad en México, por lo que es fundamental promover un modelo de gobernanza que promueva el PSTPS generando alternativas de empleo.

Así, partiendo de un enfoque cualitativo, aplicando una metodología hipotético-deductiva bajo un diseño descriptivo, causal, exploratorio y no experimental, con un horizonte de tiempo transversal simple, se abordan los elementos de la gestión de la gobernanza (visión, coordinación, planificación, evaluación, monitoreo), así como los alcances del PSTPS, cuyos indicadores de contrastación parten de la información publicada por fuentes oficiales como son: el Instituto Mexicano del Seguro Social (2020), Instituto Nacional de Estadística y Geografía (2018) y la Secretaría del Trabajo Previsión Social (2020).

\section{Marco conceptual: La gobernanza, política laboral e inclusión}

El enfoque de gobernanza es post-gubernamental (Aguilar, 2007) y apunta a la necesidad o conveniencia de un nuevo proceso directivo de la sociedad, en el cual predominen una integración de las agendas laboral, social, económica y de salud, bajo un modelo de coordinación de políticas para el desarrollo.

La crisis y/o colapso fiscal o económico o político de los estados sociales en los años ochenta y noventa |ocasionó daños y costos sociales y no sólo impulsar y sostener los beneficios del desarrollo y del bienestar (Aguilar, 2007). Por tanto, el problema no es ya el gobierno, en el sentido de gobernante, sino el gobierno, en el sentido de la acción de gobernar, y ello se debe, entre otras razones, a que el gobernar incluye relación con los ciudadanos (agentes sociales, económicos, políticos) y la relevancia de una eficaz integración de sus agendas para el desarrollo. En este caso, la integración de las agendas social y laboral bajo una gobernanza para el desarrollo resulta estratégico para legitimar el papel del Estado.

El modelo de gobernanza de resultados para el desarrollo (GpRD) se concibe importante porque permite conceptualizar teórica y metodológicamente el papel del Estado mexicano para una agenda para desarrollo (Martins y Marini, 2010), y el resto de los elementos que la integran: visión, dirección, liderazgo, estrategias transversales, capacitación, incentivos, coordinación, implementación, monitoreo, evaluación, planeación y rediseño de políticas respectivamente.

La coyuntura actual asociada a la pandemia del Covid-19 ha implicado una demanda creciente de un Estado más efectivo, eficiente y transparente, particularmente porque han aumentado las demandas sociales. En consecuencia, se requiere un Estado de Bienestar con base en un nuevo pacto social (fiscal, social y laboral) que incluya la universalización de acceso a los derechos, ingreso básico universal y alternativas laborales para los grupos vulnerables. La viabilidad de tal propuesta está asociada con fortalecer 
las capacidades de gestión financiera institucional, que permitan promover una política laboral que incida en la generación de empleos para los grupos vulnerables.

El argumento para plantear es que tales elementos han estado presentes en las mejores políticas laborales en el ámbito internacional. Por lo cual, es fundamental concebir una eficaz gobernanza de esa transversalidad para el fomento de empleo y mejores condiciones.

Las políticas públicas para promover empleos se asocian a la forma en que se entiende el "valor". El reto gubernamental es cómo generar ese valor. $\mathrm{Y}$ en tal sentido, la gobernanza crea un valor público (Moore, 2011) con base de identificar oportunidades, alternativas, fortalezas, capacidades y en un contexto de pandemia, según la resiliencia institucional. La gobernanza puede crecer la economía con base de empleos para los distintos estratos, promueve la innovación y contribuye a una menor desigualdad, si y solo si se fundamenta en una eficaz institucionalidad: de su gestión, de sus redes de gobernanza multinivel, de su sentido de responsabilidad, de la certeza del Estado de derecho y de sus valores tradicionales: transparencia, rendición de cuentas y legalidad. De esta manera, se revitaliza el debate del valor y de su impacto en la generación de empleo, apoyando el propósito público y el desarrollo humano.

Otra forma de promover el desarrollo humano a través de la generación de empleos es en la formulación de políticas. El Estado tiene un papel empresarial clave para formar mercados y diseñando políticas que aborden grandes desafíos y creen valor público (Mazzucato, 2020). Los mercados solos, por ejemplo, no abordarán el cambio climático o la desigualdad. Le concederán prioridad en la agenda de políticas en la medida es que se concibe un valor, un impacto social, y que genera beneficios transversales en materia de empleo a través de una política laboral.

Desde la perspectiva de Mazzucato (2020), los Estados deben aprovechar estos "problemas perversos" como oportunidades para establecer misiones audaces y ambiciosas hacia las cuales las políticas, sectores y financiamiento están orientados a cumplir, por ejemplo, la generación de empleo. En tal sentido, un desafío es adoptar nuevos métodos de presupuesto basados en resultados. Dado que la preocupación institucional en contexto de escasez y altas demandas sociales es la creación del valor social. En consecuencia, se asume la importancia de la integración entre papeles del Estado, gerencia financiera, oportunidad, valor e impacto en la generación de empleos con opciones de innovación.

Siguiendo a Mazuccato (2020), las misiones ecológicas pueden dar forma al crecimiento económico en una dirección sostenible que genere empleos, mejoras en los niveles de vida y desarrolle la resiliencia. Esas misiones se crean y se institucionalizan con base de definir y hacer viable el modelo de gobernanza, las prioridades, la agenda, las estratégicas y sus prioridades. Con ello, se fundamenta una política laboral inclusiva, la cual se fundamenta con base en una adecuada formulación de alternativas, su viabilidad y su impacto en la generación de empleos diversos, según articulación oferta y demanda laboral.

Una visión poco proactiva de la gestión ha condicionado los procesos de resiliencia institucional tanto, para abordar la crisis y los efectos laborales de la pandemia. Situación que se agudiza en países en desarrollo, por la dificultad de conciliar la gobernanza para la eficacia gubernamental, la responsabilidad institucional para la creación de valor y la priorización de una política de empleo como parte de la política de desarrollo. Por ello, es esencial promover una capacidad gubernamental articulada que permite fomentar una gestión eficaz de las causas, impactos y alternativas de política laboral.

La capacidad gubernamental implica generar empleos con base de decisiones políticas informadas e inteligentes y que generan valor. Esos componentes son determinantes para una implementación 
eficaz de una política laboral, según las prioridades de los Objetivos del Desarrollo Sustentables (ODS) de la Agenda 2030 (Naciones Unidas, 2020). Para lo cual se requiere de un modelo de gobernanza orientado a crear las competencias necesarias, en particular técnicas y profesionales, para acceder al empleo, el trabajo decente y el emprendimiento. Al igual que promover alternativas laborales que reduzcan la proporción de jóvenes que no están empleados y no cursan estudios ni reciben capacitación, pero que demandan opciones laborales, con la finalidad de reducir su vulnerabilidad y con ello mejorar su nivel de vida. Para ello es fundamental articular las políticas nacionales con la estrategia mundial para el empleo de los jóvenes y aplicar el Pacto Mundial para el Empleo promovido por la Organización Internacional del Trabajo.

Para ello, los gobiernos deben de contar con la capacidad para promover procesos de planificación estratégica en la política laboral según las siguientes vertientes:

- Conocimientos técnicos y empleabilidad

- Empleo juvenil

- Políticas activas de mercado de trabajo

- Crecimiento económico y generación de empleo

- Igualdad de género y no discriminación

- Políticas nacionales de empleo

- Protección social

- El futuro de trabajo

Estas dimensiones se tienen que gestionar con base de un modelo de gobernanza orientado a generar impactos en términos de valor e inclusión. En este contexto, el aporte de la gobernanza radica en que propone un marco conceptual y metodológico para promover la interacción, la cooperación y/o la asociación entre los distintos sectores gubernamentales y sociales (Kooiman, 2010). Para ello se proponen diversos elementos de gestión que permiten una operacionalización e institucionalización eficaz de una política integral en materia de empleo. Este modelo de gobernanza es estratégico, porque ejecuta su paradigma según contexto y capacidades presentes y acorde con la problemática laboral.

\subsection{Contexto de la problemática laboral}

Según la Comisión Económica para América Latina y el Caribe (2020), habría 11,6 millones de desocupados más que en 2019, lo que impactará en una reducción de los ingresos laborales de las personas y de los hogares. La pérdida de ingresos afectará a quienes se encuentran en situación de pobreza y vulnerabilidad y quienes están precariedad laboral, ya que la imposibilidad de trabajar desde el domicilio, las condiciones de hacinamiento y la falta de acceso a agua y saneamiento aumentan el riesgo de infección de la población en situación de pobreza y vulnerabilidad. Desde la perspectiva de CONEVAL, México enfrenta esta contingencia en condiciones de vulnerabilidad debido, entre otros elementos, a altas prevalencias de diabetes y enfermedades cardiovasculares; precariedad laboral y estancamiento económico; carencias en el acceso al agua y hacinamiento, y brechas en el cumplimiento de los derechos sociales: salud, alimentación, educación, seguridad social y vivienda. Este contexto socio laboral y sanitario condicionará la agenda post 
Covid-19, lo que implicará el reto de una mayor capacidad del Estado mexicano para prever un posible rebote de contagios y evitar reducir los logros alcanzados en materia de política social (CONEVAL, 2020).

Según el propio CONEVAL (2020), entre 2008 y 2018 el porcentaje de población en situación de pobreza disminuyó 2.5 puntos porcentuales, al pasar de 44.4 por ciento a 41.9 por ciento, lo que representa una reducción media anual de 0.24 puntos porcentuales. La población en situación de pobreza extrema descendió de 11.0 por ciento a 7.4 por ciento, es decir, de 12.3 a 9.3 millones de personas. Así mismo, la información generada por CONEVAL (2020) también muestra que, entre 2008 y 2018, se registró una mejora en los indicadores de carencias sociales a nivel nacional y en la mayoría de las entidades federativas; las carencias por acceso a los servicios de salud, así como por calidad y espacios de la vivienda tuvieron las reducciones más amplias durante este periodo. Esta disminución de la pobreza es importante, pero ha sido insuficiente para reducir los enormes rezagos sociales y en especial el impacto de los grupos sociales más vulnerables, los cuales con el Covid-19 serán los más afectados. Estos rezagos estructurales en materia de política social en México son previos al Covid-19 y el mal desempeño del presupuesto social, en especial porque ha sido un gasto que no ha impactado en otras dimensiones (educación, capacitación, control de la corrupción, mejora salarial). De ahí la importancia de promover una eficaz gobernanza multidimensional y multinivel para el desarrollo.

Los escenarios de dicho organismo, según efectos del Covid-19 para el caso mexicano son los siguientes: el total de personas en situación de pobreza por ingresos (las personas cuyo ingreso es insuficiente para adquirir una canasta alimentaria, bienes y servicios básicos) se incrementa, entre 7.2 y 7.9 puntos porcentuales (entre 8.9 y 9.8 millones de personas). El número total de personas en situación de pobreza extrema por ingresos (las personas cuyo ingreso es insuficiente para adquirir una canasta alimentaria) se incrementa entre 4.9 y 8.5 puntos porcentuales ( 6.1 y 10.7 millones de personas). En suma, la crisis podría provocar que la población que en 2018 no era pobre ni vulnerable, tenga afectaciones que los lleven a encontrarse en alguna de estas condiciones. De ahí la relevancia de impulsar una eficaz gobernanza para el desarrollo, en donde se articulen las políticas laborales, social y con impactos en la creación de empleos para grupos vulnerables (CONEVAL, 2020).

La Población Económicamente Activa (PEA) en México durante el primer trimestre de 2019 se conformaba por 56,038,471 personas, cifra que se modificará para 2020, destacando que en período comprendido de enero a marzo, la población total presentaba una configuración poblacional de 126,661,703 personas, de las cuales el 76 \% estaban en edad de trabajar (15 años y más), sin embargo la PEA, se elevó a $57,328,364$ personas, de los cuales el $39.5 \%$ son mujeres y el $60.5 \%$ son hombres (ver Tabla 1 ) (Secretaría del Trabajo y Previsión Social, 2020).

Tabla 1. Configuración de la PEA en México al primer trimestre de 2019 y 2020

\begin{tabular}{cccccc} 
& Población & Pea & Ocupados & Hombres & Mujeres \\
\hline 2019 & $125,489,149$ & $56,038,471$ & $54,152,266$ & $32,762,121$ & $21,390,145$ \\
2020 & $126,661,703$ & $57,328,364$ & $55,352,304$ & $33,484,209$ & $21,868,095$ \\
\hline
\end{tabular}

Fuente: Elaboración propia con base en el Instituto Nacional de Estadística, Geografía en Informática (2020) y la Secretaría del Trabajo y Previsión Social (2020). 
No obstante, para ese último período el empleo informal o subocupación laboral a nivel nacional se integraba por 31.1 millones de personas (Instituto Nacional de Estadística, Geografía e Informática, 2020), condición que las ubica como un grupo en situación de vulnerabilidad laboral, toda vez que se caracterizan por su precariedad, con sueldos menores al mínimo y horarios extenuantes. Además, carecer principalmente de certezas jurídicas y prestaciones laborales como el acceso a la seguridad social (Comisión Nacional de los Derechos Humanos, 2020). Estos temas críticos se pretenden erradicar con implementación del programa sectorial laboral de la presente administración federal. Lo que implica el reto de una eficaz implementación de un modelo de gobernanza con base de sus distintos elementos de gestión (visión, coordinación, incentivos, agenda, planificación, evaluación y monitoreo).

Entre los principales retos de la política laboral existente destaca el combate a la precariedad del trabajo, donde una de sus principales manifestaciones es la informalidad laboral que se caracteriza por la ausencia de protección desde los esquemas normativos (Instituto Nacional de Estadística, Geografía e Informática, 2018); de ahí que destaquen cuatro elementos fundamentales, como la democratización de la productividad y conservar la paz laboral, pero sobre todo, impulsar la creación de empleos formales de calidad y salvaguardar los derechos de los trabajadores, retos que prevalecen desde sexenios anteriores (Gobierno de México, 2020; Gobierno de la República, 2013; Secretaría del Trabajo y Previsión Social, 2020). El tema central es cuál es viabilidad de lograr estos objetivos, si son problemas estructurales en la política laboral mexicana, lo que refleja la ausencia de una eficaz política laboral con valor público y una efectiva gobernanza multidimensional para el desarrollo.

Así la población ocupada en esquemas de vulnerabilidad laboral, cuya dependencia o vínculo no es reconocido legalmente por su fuente de trabajo es conformada por 28.7 millones de personas, de las cuales 10 millones son mujeres (ver Figura 1), que trabajan en condiciones de informalidad, sin contar lo ningún tipo de protección relativa a la seguridad social (Instituto Nacional de Estadística, Geografía e Informática (2018).

Figura 1. Población en ocupación informal al mes de agosto de 2019 y 2020

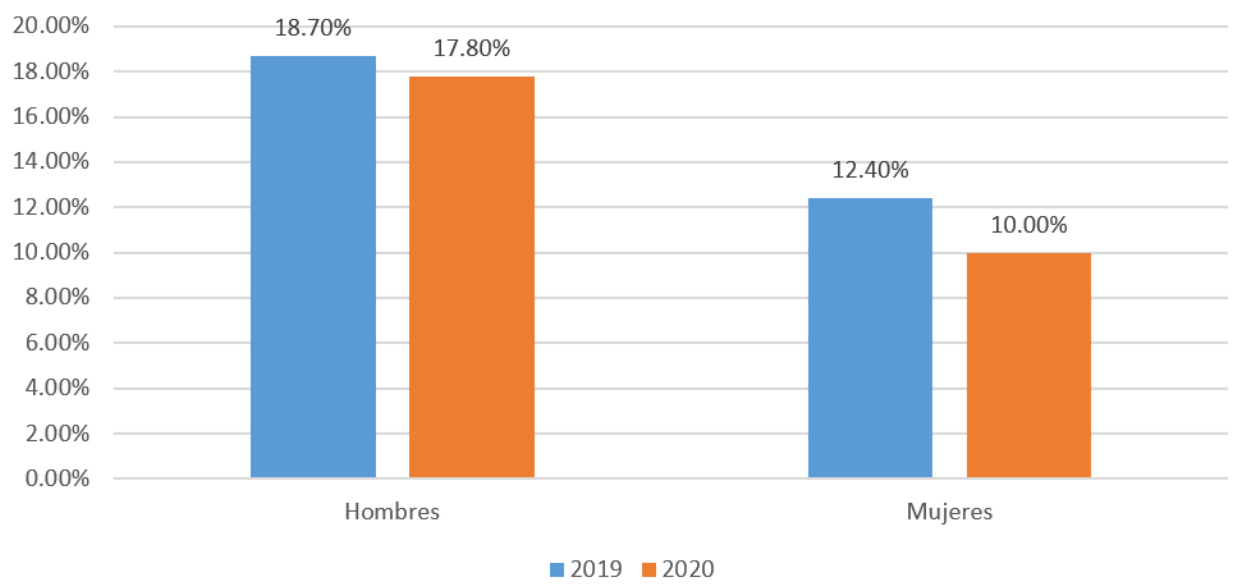

Fuente: Elaboración propia con base en el Instituto Nacional de Estadística, Geografía e Informática (2018). 
Por otra parte, desde hace décadas se ha reconocido que la región ubicada en la frontera norte de México, presenta características y problemáticas particulares, destacando el rápido crecimiento de las principales ciudades fronterizas, la importante captación de divisas y un alto potencial económico (Stern, 1967; Urquidi y Sofía, 1975); además, estudios recientes (Gómez y Hernández, 2016) ubican dicha zona como una de las más competitivas de la República Mexicana, derivado de que México y Estados Unidos comparten una frontera que se extiende a lo largo de 3,175 kilómetros, conformada por 57 localidades fronterizas, en diez estados, cuatro del lado americano: California, Arizona, Nuevo México y Texas, y seis del lado mexicano: Baja California Norte, Coahuila, Nuevo León, Sonora, Tamaulipas y Chihuahua (Secretaría de Relaciones Exteriores, 2020). De este contexto fronterizo y transfronterizo, tales como la Conferencia de Gobernadores Fronterizos, se identifican alternativas de generación de empleo para el bienestar. Sin embargo, las asimetrías entre las entidades fronterizas no abonan a promover la cooperación en materia de opciones laborales para los grupos vulnerables en las entidades mexicanos. La tendencia central es la reproducción de la política de integración laboral vía los trabajadores transmigrantes o mediante los empleos generados por la industria maquiladora, que abonan a la integración de las cadenas de valor.

Si bien la línea divisoria impone condiciones asimétricas de administración y control propios de cada país, las comunidades que la conforman, mantienen una problemática común, binacional y regional, resultado de un largo proceso histórico, fuertemente integradas económica y comercialmente, además de un importante crecimiento económico, especialmente para el Estado Mexicano (Secretaría de Relaciones Exteriores, 2018), de ahí que para el año de 2018 los seis estados que conforman dicha región, generaron una participación acumulada del 12.2 \%, del Producto Interno Bruto de México, cuya aportación principal deriva de las actividades relativas al sector terciario (ver Tabla 2), de acuerdo al Instituto Nacional de Estadística, Geografía e Historia (2019).

Tabla 2. Aportación al Producto Interno Bruto Nacional de las Entidades de la Frontera Norte de México

\begin{tabular}{ccccc}
\hline Entidad & Aportación total & Primarias & Secundarias & Terciarias \\
\hline Baja California & 2.3 & 1.8 & 3.89 & 1.4 \\
Sonora & 1.2 & 1.2 & 0.5 & 2.6 \\
Chihuahua & 1.6 & 3.3 & -0.6 & 3.1 \\
Coahuila & 1.3 & 5.4 & -0.3 & 3 \\
Nuevo León & 3.6 & 0.6 & 4 & 3.4 \\
Tamaulipas & 2.2 & -6.8 & 4 & 1.7 \\
Total & 12.2 & 5.5 & 11.49 & 15.2 \\
\hline
\end{tabular}

Fuente: Elaboración propia con base en Instituto Nacional de Estadística, Geografía e Historia (2019).

Sin embargo, otras fuentes (Impacto Noticias, 2020; Tiempo, 2020) refieren que las entidades fronterizas del norte del país son las que más aportan al PIB nacional, destacando que Chihuahua, Baja California y Sonora aportan un $3.4 \%$ cada uno, en tanto que Nuevo León es el tercer estado aportando el 7.9 $\%$, logrando con ello un valor acumulado superior al $18 \%$. 
Lo anterior es consecuencia, entre otras cosas, de las tasas de participación de la PEA que presenta dicha región, cuya cifra más representativa se identifica en el Estado de Chihuahua con un 62,8 \%, en tanto que las tasas de desocupación corresponde al Estado de Coahuila (ver Tabla 3).

Tabla 3. Tasas de participación de la PEA y la desocupación al primer trimestre de 2020

\begin{tabular}{ccc}
\hline & Tasa neta de participación PEA & Tasa de desocupación \\
\hline Media Nacional & 59.9 & 3.4 \\
Baja California & 61.3 & 2.3 \\
Sonora & 62.8 & 4.5 \\
Chihuahua & 61.3 & 3.2 \\
Coahuila & 59.0 & 4.8 \\
Nuevo León & 60.9 & 3.9 \\
Tamaulipas & 60.1 & 3.1 \\
\hline
\end{tabular}

Fuente: Elaboración propia a partir del Instituto Nacional de Estadística, Geografía e Informática (2020) y la Secretaría del Trabajo y Previsión Social (2020).

Uno de los principales elementos desde los cuales es posible abordar los aspectos señalados previamente, lo constituye el número de empleos formales registrados ante el Instituto Mexicano del Seguro Social (Instituto Mexicano del Seguro Social, 2020) y que para efectos del presente artículo, se observaron los indicadores relativos al año de 2019 y el primer trimestre de 2020, desde cuya configuración por entidad federativa, destaca la contribución del estado de Nuevo León (ver Figura 2).

Figura 2. Configuración de Trabajos Formales por entidad federativa

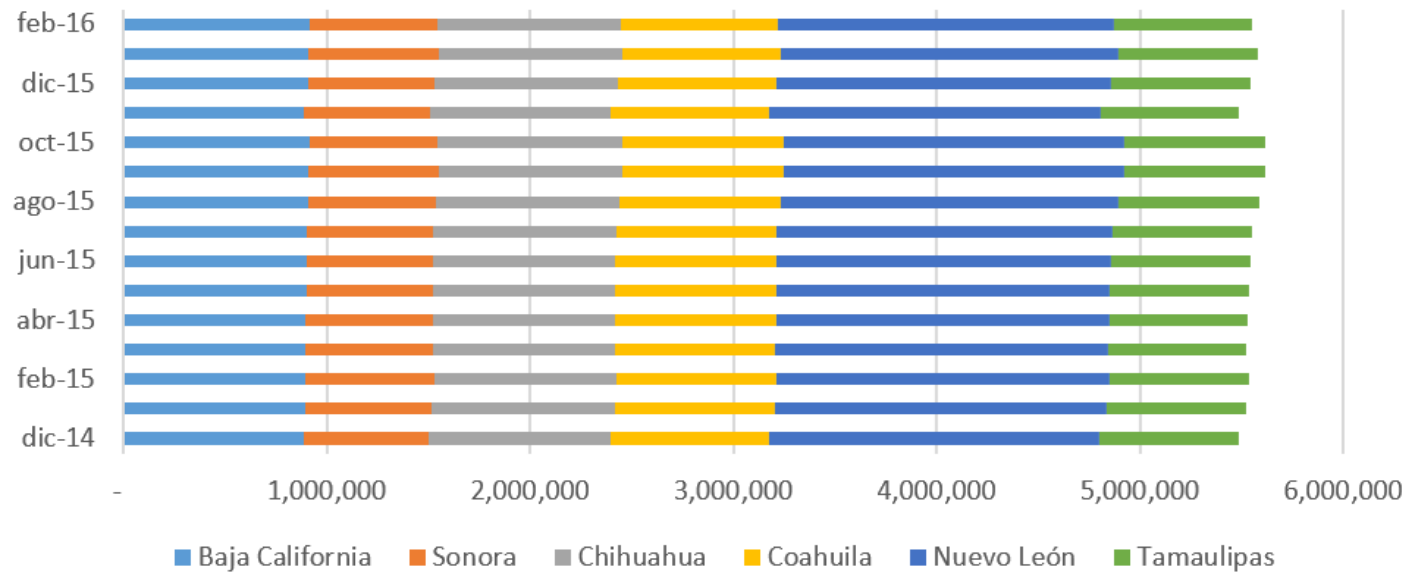

Fuente: Elaboración propia con base en el Instituto Mexicano del Seguro Social (2020). 
Para el mes de julio de 2020, se identifica que en el $35.2 \%$ de los hogares mexicanos reportan que durante el período de la fase 3, alguno de sus integrantes perdió su trabajo o fuente de ingresos durante los cuatro meses anteriores (Instituto de Investigación para el Desarrollo con Equidad, 2020); además, las cifras de empleos formales registrados ante el Instituto Mexicano del Seguro Social refleja que durante los meses de marzo a julio de 2020, se perdieron más de 1'300,000 empleos formales, lo que constituye una reducción del $5.5 \%$ en comparación con las cifras de febrero del mismo año (Instituto Mexicano del Seguro Social, 2020). Por otra parte, fuentes no oficiales refieren a partir de la suspensión de actividades no esenciales y la falta de movilidad social por COVID-19, se perdieron más de 12 millones de espacios laborales en ámbitos formales, derivando en una de las peores crisis laborales en décadas (Sinembargo, 2020). De ahí la importancia de analizar si el Programa Sectorial Laboral es una opción viable para generar los empleos perdidos y con ello reducir el desempleo, subempleo y la precariedad laboral.

Para el segundo trimestre de 2020, el Producto Interno Bruto presento una reducción de - 18.7 \% (Instituto Nacional de Estadística, Geografía e Informática, 2020), cifra que finales del mes de octubre de 2020, reportaba una nueva disminución del -9.8\% el PIB, aunado al cierre de más de 150,000 pequeños negocios, de los cuales solo 8,000 eran pequeños comercios ubicados en el estado de Nuevo León, y las que sobreviven refieren tener ventas inferiores al 50 \% (Alianza Nacional de Pequeños Comerciantes, 2020) .

La participación de las mujeres en las actividades económicas en América latina es muy importante, no solo realizan aquellas relativas al cuidado familiar no remuneradas, sino que se han incorporado en ámbitos laborales diversos, como la industria manufacturera o ensambladora de exportación, en cadenas de subcontratación y en distintas ramas del sector terciario, donde la segregación ocupacional por sexo se traduce en discriminación salarial y reducidas posibilidades de movilidad social (Oliveira y Ariza, 2000). En las sociedades predominantemente industriales, los esquemas de producción se configuran de manera disociada del ámbito doméstico, donde la incorporación de las mujeres al mercado laboral como manifestación aparente de igualdad de oportunidades entre hombres y mujeres, se traduce generalmente en una doble jornada para las últimas, ya que no se les exime de cumplir con los roles preponderantemente femeninos (Alameda, 2017).

En México, la configuración de la fuerza laboral de las mujeres, se caracteriza por mantener un esquema diferenciado respecto al de los hombres (Maldonado, 2010), integrando procesos de informalización, terciarización del mercado de trabajo, su descalificación y desalarización, además de estar insertas principalmente en actividades menos calificadas (Mancini, 2016), intensificándose con ello la diversidad de problemas relativos, donde destacan la precariedad y la inseguridad laboral (Ariza, 2016; Martínez, Marroquín y Ríos, 2019).

Si bien se observa un incremento en las oportunidades laborales para las mujeres y una disminución importante en la brecha educativa frente a los hombres, aún prevalecen las desigualdades que vulneran los derechos de las mujeres, convirtiéndolas en un grupo en situación de vulnerabilidad, mismo que experimenta distintas formas de violencia, de entre las cuales destaca que el $42.4 \%$ de ellas presentan una condición de pobreza (CONEVAL, 2020). 
Figura 3. Porcentaje de mujeres en situación de pobreza en los estados del norte de México

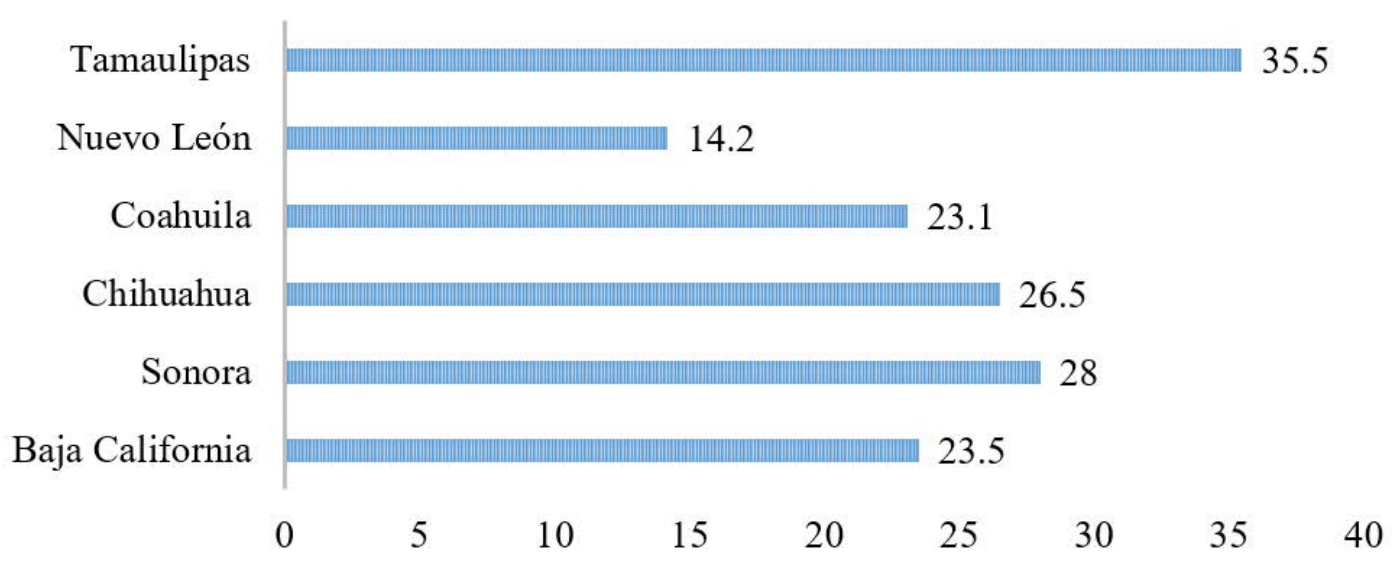

Fuente: Elaboración propia con base en CONEVAL (2020).

Lo anterior cobrará especial relevancia considerando que muchos hogares mexicanos han logrado enfrentar los impactos económicos a partir de los ingresos provenientes de la mano de obra femenina, en mercados de trabajo e ingresos disímiles (Alarcón y Mckinley, 1998; Cortés, 2000), además de registrarse que del $30 \%$ de las mujeres trabajadores, refieren haber experimentado con ello desigualdad, disparidad en carga de trabajo no remunerado, violencia y/o discriminación, derivando principalmente en condiciones de pobreza y pobreza extrema (CONEVAL, 2020; Instituto Nacional de Estadística, Geografía e Informática, 2017), circunstancias que se caracterizan por carencias o necesidades básicas insatisfechas, vinculadas a la calidad o cantidad en la ingesta de alimentos, acceso limitado o nulo a los servicios de salud, educación y vivienda, derivadas de ingresos insuficientes (García y Zapata, 2012).

\section{Programa Sectorial de Trabajo y Previsión Social 2020-2024}

Como se aprecia, la vulnerabilidad y la precariedad laboral se agudiza en las mujeres, lo que aunado a la problemática estructural de discriminación, abona a reproducir tal contexto estructural. De ahí la cuestión de que, si el Programa Sectorial de Trabajo y Previsión Social (PSTPS) disminuirá tales problemas, con base de políticas inclusivas y de cohesión social y que generen valor público (ver Tabla 4).

Tabla 4. Algunos elementos del Programa Sectorial de Trabajo y Previsión Social 2020-2024

Objetivos prioritarios del Programa Sectorial de Trabajo y Previsión Social 2020-2024

1.- Lograr la inclusión de jóvenes a través de la capacitación en el trabajo

2.- Impulsar el diálogo social, la democracia sindical y la negociación colectiva auténtica conforme al nuevo modelo laboral 
Rua

9 Gobernanza y Política Laboral en México... / Vol. 13, No. 22, enero-junio 2021

DOI: http://dx.doi.org/10.20983/novarua.2021.22.1

3.- Recuperar el poder adquisitivo de los salarios mínimos y los ingresos para mejorar la calidad de vida de las y los trabajadores

4.- Dignificar el trabajo y estimular la productividad mediante la vigilancia al cumplimiento de la normativa laboral

5.- Lograr la inserción en un empleo formal de las personas desempleadas, trabajadores en condiciones críticas de ocupación y personas inactivas con disponibilidad para trabajar, con atención preferencial a quienes enfrentan barreras de acceso a un empleo formal

Fuente: Elaboración propia con base en la Secretaría del Trabajo y Previsión Social (2020).

El primer objetivo de la Secretaría del Trabajo y Previsión Social es la inclusión de los jóvenes; la propuesta gubernamental es que 2.3 millones de jóvenes que no desempeñan alguna actividad, obtengan una beca para capacitarse en el trabajo hasta por doce meses tanto en empresas, talleres, negocios, comercios, como en organizaciones sociales e instituciones públicas (Secretaría del Trabajo y Previsión Social, 2020). El Programa Jóvenes Construyendo el Futuro es una alianza entre el sector público, privado y social, con su implementación al finalizar 2019, alrededor de un millón de jóvenes ya habrán comenzado su capacitación para su ulterior inserción al sector laboral. Entre los retos del mencionado Programa están que puedan integrar a una mayor cantidad de jóvenes, considerando la problemática del tejido social y de sus riesgos. Con base en las restricciones presupuestarias, es fundamental una eficaz coordinación interinstitucional entre los diferentes programas gubernamentales en materia laboral (Gobierno de Mexico, 2020).

Un objetivo más del Programa Sectorial es fortalecer las políticas activas del mercado de trabajo. Con ello se pretende corregir las desigualdades en el acceso a la información en el mercado laboral, aprovechar las nuevas tecnologías para hacer más eficiente la intermediación entre los buscadores de empleo y las vacantes ofertadas, dar asesoría y orientación vocacional; optimizar los programas de movilidad laboral dentro de México y hacia otros países, y ofrecer cursos de capacitación (Gobierno de México, 2020). En especial los programas de capacitación son una alternativa para fortalecer las capacidades de las personas en situación de desempleo o de precarización laboral. El reto es la cantidad de cursos, su pertinencia y el impacto en la inserción laboral en un contexto de restricciones de generación de empleo, por los efectos de la pandemia.

El PSTPS 2020-2024 destaca que la juventud en México ha sido un sector de la población que durante las administraciones pasadas fue olvidado. La falta de oportunidades, derechos y políticas públicas para el desarrollo de los jóvenes en México provocó una ruptura en el tejido social que ha derivado, entre otras problemáticas, en desempleo, crisis de violencia e inseguridad. Por ello se cuestiona si el Programa Sectorial tendrá la capacidad conceptual, metodológica y de inserción laboral, según los problemas estructurales que han caracterizado el empleo de los jóvenes en México. Ante las dificultades de inserción laboral de los jóvenes, es fundamental un modelo de gobernanza de la política laboral con un enfoque multidimensional y multinivel, que permita optimizar los recursos de los diferentes niveles de gobierno, bajo una efectiva coordinación interinstitucional.

Según el PSTPS 2020-2024 el bono demográfico que se perfilaba como una gran oportunidad para el desarrollo del país, no fue aprovechado por las administraciones anteriores. Los jóvenes entre 18 y 29 años suman 24.3 millones de personas, de las cuales 19.1 millones habitan en zonas urbanas y 5.2 millones en 
zonas rurales. El 61.7\% de estos jóvenes forman parte de la PEA y el 38.3\% pertenecen a la Población No Económicamente Activa. La PEA cuenta con una mayor proporción de hombres (62.1\%), mientras que las mujeres son mayoría en la Población Económicamente Activa (72\%). No obstante, es necesario destacar que dentro de la PEA se identifican 937,378 jóvenes "desocupados"; es decir, que buscaron un empleo la semana anterior a la aplicación de la ENOE 2018 y no encontraron. Estas restricciones del mercado laboral indicadas por el PSTPS 2020-2024 reflejan la dificultad de la inserción laboral en contextos de contracción económica y que se agudizaran por los efectos de la pandemia. Por ello se reitera en la relevancia de una eficaz coordinación interinstitucional de los programas generadores de empleo.

Acorde con el PSTPS 2020-2024 una parte importante de las oportunidades de trabajo para los jóvenes han surgido en el sector informal de la economía, donde la remuneración es más baja que en el sector formal. Entre 2005 y 2017, el sector informal fue el mayor empleador de jóvenes. Durante ese mismo período, la tasa de informalidad entre los jóvenes (62.4\%) ha sido mayor que la de las personas mayores de 30 años (56.8\%) (6). Esto provoca que una proporción considerable de los jóvenes mexicanos no tenga acceso a seguridad social. Este es un problema estructural, que se ha agudizado en el transcurso de los años. Por ello, se reitera que, ante las dificultades de inserción laboral de los jóvenes, es fundamental que el PSTPS tenga un modelo de gobernanza de la política laboral con un enfoque multidimensional y multinivel, que permita optimizar los recursos de los diferentes niveles de gobierno, bajo una eficaz coordinación interinstitucional.

La precariedad laboral de los jóvenes, también se asocia en el caso mexicano con el bajo nivel educativo y la deserción escolar; según datos de la OCDE, solamente $17 \%$ de los mexicanos entre 25 y 64 años cuentan con un título de educación superior, frente al promedio de $37 \%$ de los demás países que conforman a tal organización (Gobierno de Mexico, 2020).

Según el propio PSTPS 2020-2024 se estima que la mitad de las empresas mexicanas afirman tener dificultades para encontrar personal capacitado para los puestos de trabajo que ofertan. Esta situación implica que debe ser analizado el mecanismo de reclutamiento por parte de los empleadores, la interacción entre los sistemas educativo y productivo, así como seguir fomentando mecanismos de capacitación que permitan una mayor inclusión al mercado laboral. Estas políticas son parte de la gestión de la transversalidad del Programa Sectorial e implican fortalecer su viabilidad, en un marco en que la pandemia del Covid-19 ha generado mayor desigualdad y precarización laboral.

Según el PSTPS 2020-2024, una de las consecuencias de la disfuncionalidad del mercado laboral es que los trabajadores quedan atrapados en un círculo vicioso en el que solo consiguen empleos informales, que no les permiten mejorar sus competencias laborales, lo que les dificulta acceder posteriormente a empleos formales y tener una trayectoria laboral ascendente. La informalidad laboral sigue siendo muy elevada, $57 \%$ a nivel nacional a fines de 2018 , aunque con grandes diferencias por entidad federativa. Este es otro de los problemas estructurales de la política laboral mexicana. El diagnóstico es preciso. El desafío de la política es cuál es la viabilidad de reducir tal círculo vicioso, con políticas integrales orientadas a disminuir dichos problemas con un enfoque multinivel y de transversalidad.

La propuesta conceptual para reducir las limitaciones del diagnóstico y promover los objetivos del PSTPS es el modelo de gobernanza para resultados, según los siguientes componentes: 
-Visión hacia la creación de empleos, según la diversidad de demandas y reducción de las limitaciones del diagnóstico del PSTPS.

- Evaluación estratégica de las capacidades institucionales actuales para generar empleo, según las restricciones financieras, económicas, sociales y presupuestarias.

- Definición de prioridades de las acciones y estrategias focalizadas en materia de generación de empleo, según pertinencia e impacto laboral.

- Liderazgo estratégico e interinstitucional para promover empleo, con base de una política integral y según demandas por nivel de vulnerabilidad.

- Coordinación intraorganizacional e interinstitucional de una política laboral, con base de una agenda multinivel y multiactor.

- Estrategias transversales para el fomento de una política laboral, se implementan diversas estrategias en distintas dimensiones (social, educativa, legal, género, financiera, institucional). - Implementación transversal de políticas para el empleo, ejecución de diversas políticas, según prioridades del PSTPS.

- Monitoreo de las políticas transversales para la generación de empleo, seguimiento integral de las políticas verificando logros y resultados.

- Evaluación de los logros e impactos de la política laboral.

- Indicadores de gestión de la política de empleo.

Se considera que la ausencia de tales elementos de gestión son determinantes para promover una eficaz, efectiva y eficiente política integral de empleo, particularmente en un contexto de restricciones. Dichos aspectos se resumen la Tabla 5:

Tabla 5. Gobernanza de Empleo y Gobernanza Reactiva

\begin{tabular}{cc} 
Eficaz gobernanza de empleo & Gobernanza reactiva \\
\hline Visión estratégica y transversal & Visión reactiva \\
Dirección al resultado y valor & Dirección convencional no crea valor público \\
Liderazgo estratégico y colaborativo & Ausencia de liderazgo \\
Eficaz coordinación interinstitutional & Coordinación limitada \\
Agenda de prioridades, según vulnerabilidad & Agenda general \\
Planificación estratégica en distintos plazos & Limitada planificación \\
Evaluación estratégica & Limitada evaluación transversal \\
Monitoreo permanente & Limitado monitoreo \\
Indicadores de eficacia y de impacto social & Indicadores de eficiencia \\
\hline
\end{tabular}

Fuente: Elaboración propia con base de Martins y Marini (2010).

Desde esta perspectiva, el concepto de gobernanza está relacionado con las capacidades institucionales y su viabilidad para implementar efectivas políticas transversales para la generación de diversas opciones de empleo. El fin es fortalecer las capacidades de planificación, monitoreo, seguimiento, evaluación y gestión para una efectiva política laboral. 


\section{Estado de Bienestar, algunos retos en contexto de Covid-19 y su viabilidad}

La construcción de un Estado de bienestar y sistemas de protección social universal son clave para evitar otra década perdida en México, como opciones para moderar los efectos de la crisis en las economías, frenar el aumento del desempleo y la pobreza. En ese marco, es fundamental el fortalecimiento de la institucionalidad social para implementar políticas sociales responsables y bajo una eficaz alianza público-privada en pro de la generación de valor y opciones laborales, en especial en los grupos vulnerables. Para la planificación, diseño e implementación de medidas de protección social es importante proteger el gasto público social y contar con sistemas de información, seguimiento y evaluación de las prestaciones sociales, incluidos registros de la población destinataria o potencialmente destinataria que sean lo más amplios y actualizados posible (CONEVAL. 2020), es decir, es necesaria la articulación entre la política, la gestión y los procesos de evaluación como parte del modelo de GpR con valor público y según las prioridades de la demanda de empleo.

En este marco conceptual es fundamental una eficaz coordinación, cooperación y planificación estratégica interinstitucional. Acorde con el CONEVAL (2019), se requieren medidas coordinadas de los tres órdenes de gobierno para promover que los hogares que tendrán dificultades en el ingreso puedan recibir subsidios temporales en el costo de los servicios básicos de la vivienda. Asimismo, se debe asegurar la adecuada operación de los programas federales que realizan transferencias monetarias directas (CONEVAL, 2020). Fortaleciendo la transparencia, rendición de cuentas y promoviendo alternativas laborales, por ejemplo, las microempresas con sentido comunitario y para la inclusión social. En estas acciones se puede apreciar el aporte de la GpR, porque integra los indicadores y plantea una estrategia de gestión de acuerdo con una agenda, prioridades, disponibilidad de recursos, tipo de necesidades y el impacto esperado.

El país ha tenido limitaciones para crecer y disminuir los problemas sociales estructurales de pobreza, marginalidad, exclusión social y violencia (Cordera y Tello, 2010). Los gobiernos de alternancia han sido incapaces de disminuir los problemas sociales. En efecto, sigue pendiente fortalecer la racionalidad-efectividad de la acción gubernamental, con base de resultados, realizaciones, y no solo visiones y proyectos, en consecuencia, la cuestión de la efectividad directiva de los gobiernos democráticos sigue siendo una prioridad del Estado mexicano (Aguilar, 2020), en especial con respecto a la política laboral.

En efecto, la crisis de la deuda de los años ochenta llevó a incrementos muy significativos de los niveles de pobreza y desempleo. La región demoró 25 años en retornar a los niveles de pobreza previos a la crisis. Revisar estas experiencias es importante, pues el aumento de la pobreza proyectado por la CEPAL para 2020 implica un retroceso de 13 años (Comisión Económica para América Latina y el Caribe, 2020), en tal sentido, la propuesta en materia de política social y laboral de la actual administración federal consiste en impulsar una nueva vía hacia el desarrollo para el bienestar, (Gobierno de México, 2020). En esta administración, “...el Estado no será gestor de oportunidades, que es como se presentó de manera explícita la política social del régimen neoliberal. Será, en cambio, garante de derechos” (Cámara de Diputados, 2020, p. 27). Estos propósitos gubernamentales son muy loables, al igual que las prioridades 
planteadas por el desafío es su viabilidad (Aguilar, 2020), en un marco de restricciones presupuestarias, agudizamiento de los problemas y nuevas prioridades sociales en la agenda gubernamental, que se verán limitados por los efectos económicos y sociales del Covid-19.

Se plantea desde la perspectiva de la CEPAL repensar el modelo de desarrollo y consolidar las dimensiones económicas, sociales y ambientales del desarrollo sostenible según los ODS. Dichos objetivos son relevantes y necesarios, pero se considera que no existen las condiciones institucionales en el corto plazo para una orientación hacia una política multidimensional del desarrollo. Básicamente a causa de limitaciones en la profesionalización del servicio público, una escasa priorización de los ODS articulados con el PND vigente, la escasa prioridad gubernamental del sector salud y su agenda de derechos transversales; deficiente articulación gubernamental e interinstitucional en la implementación de una política de desarrollo (Subsistema Nacional de Información Demográfica y Social, 2018) y un centralismo gubernamental que no ha logrado fortalecer tendencias hacia una descentralización eficaz y que incida en procesos de competitividad y bienestar.

Ante las grandes brechas históricas que la pandemia ha agravado, la CEPAL (2020) reitera que es el momento de implementar políticas universales, redistributivas y solidarias con enfoque de derechos. Estas políticas deben de gestionarse bajo el principio de la gestión de la escasez, dada las restricciones presupuestarias y altas demandas de servicios de salud.

Adicionalmente es fundamental incluir el criterio de viabilidad que permita la implementación en función de su pertinencia, demanda e impacto social, lo que se puede lograr con una efectividad directiva (Aguilar, 2007). Particularmente considerando la complejidad de implementar una política laboral en un contexto de pandemia, según las restricciones estructurales que han limitado la generación de empleo en grupos vulnerables. Ciertamente los proyectos de emprendimiento constituyen una respuesta orientada a coadyuvar a mejorar la condiciones socioeconómicas de la población, cuya transición hacia la viabilidad comercial requiere la articulación de todos los actores estratégicos (Zalamea y Peña, 2015); incluso a nivel mundial, el emprendimiento es considerado como un elemento fundamental para impulsar el crecimiento económico sostenible, para lo cual es necesario generar un ecosistema emprendedor desde las particularidades de cada contexto (Arenal, Armuña, Ramos y Feijoo, 2018).

Por tanto, la viabilidad de los proyectos de emprendimiento se sustenta a partir de seis elementos claves: a) Contexto político, económico, sociocultural, tecnológico y demográfico; b) Condiciones generales que influyen en el entorno empresarial: como son mercados, financieros, gestión empresarial, grados de apertura, infraestructura, mercado laboral, instituciones, legislación y tecnología $(\mathrm{I}+\mathrm{D})$; c) Condiciones del entorno empresarial específicas que influyen en la creación de nuevas empresas: infraestructura empresarial, acceso a la fuentes de financiamiento, apertura al mercado interno, infraestructura física, $\mathrm{I}+\mathrm{D}$, educación y formación, transferencia tecnológica política pública, programas públicos, cultura y valores sociales, d) Capacidad para la creación de una nueva empresa: percepción de oportunidades de negocio, motivación para crear una nueva empresa, habilidades para crear y desarrollar una nueva empresa, e) Dinámica empresarial: creación de una nueva empresa y f) Crecimiento económico nacional (Martinez, Biedma y Ruiz, 2015) observando con esto que el reto es mayúsculo. 


\section{Conclusiones}

El objetivo del artículo es analizar el aporte de la gobernanza en la promoción la eficacia del Programa Sectorial de Trabajo y Previsión Social 2020-2024 (PSTPS), en el contexto de los efectos de la pandemia del Covid-19: la desigualdad social y precariedad laboral.

El Covid-19 agudizará la desigualdad y precarización laboral que afectarán el nivel de vida de los grupos vulnerables mexicanos. Por lo cual es fundamental promover un modelo de gobernanza que promueva el PSTPS generando alternativas de empleo. Estos problemas se contemplan en el programa sectorial analizado. El tema central es cuál es viabilidad de lograr los objetivos de reducir la precariedad, bajos salarios y la informalidad laboral. Si son problemas estructurales en mercado laboral mexicano, lo que refleja la ausencia de una eficaz política con valor público y una efectiva gobernanza muldimensional y multinivel para el desarrollo.

En ese contexto, el aporte de la gobernanza puede crecer la economía con base de empleos para los distintos estratos, promueve la innovación y contribuye a una menor desigualdad, si y solo si se fundamenta en una eficaz institucionalidad: de su gestión, de sus redes de gobernanza multinivel, de su sentido de responsabilidad, de la certeza del Estado de derecho y de sus valores tradicionales: transparencia, rendición de cuentas y legalidad.

Uno de los hallazgos, es que la vulnerabilidad y la precariedad laboral se agudiza en las mujeres, lo que, aunado a la problemática estructural de discriminación, abona a reproducir tal contexto estructural. De ahí la cuestión de que si el PSTPS disminuirá tales problemas, con base de políticas inclusivas y de cohesión social. Ante las dificultades de inserción laboral de los jóvenes, es fundamental un modelo de gobernanza de la política laboral con un enfoque multidimensional y multinivel, que permita optimizar los recursos de los diferentes niveles de gobierno, bajo una efectiva coordinación interinstitucional.

En función de estos retos, se fundamentaron los desafíos del Estado mexicano en términos de su capacidad institucional para promover eficaces políticas social y laboral, según los efectos del Covid-19 y acorde a los ODS, en un marco de restricciones presupuestarias, aumento de las demandas sociales, que han agudizado las desigualdades y la precariedad laboral.

\section{Referencias}

Aguilar, L. (2007). La dimensión administrativa de la nueva gobernanza: sus prácticas y aporte. Porrúa: México.

Aguilar, L. (2020). Max Weber: modernidad y racionalidad. Recuperado de: https://www.letraslibres. com/mexico/revista/maxweber-,pdernidad-y-racionalidad.

Alameda, M. T. (2017). Emprendimiento en femenino. Dificultades de conciliación trabajo-familia y autoempleo (avances y retrocesos). En Blázquez, E. (Ed.) La negociación colectiva como vehículo para la implantación efectiva de medidas de igualdad. Madrid: Universidad Carlos III de Madrid.

Alarcón, D. y Mckinley, T. (1998). Mercados de trabajo y desigualdad de ingreso en México. Dos décadas de reestructuración. Papeles de Población, 4(18), 49-80. Recuperado de: https://www.redalyc.org/ pdf/112/11201803.pdf. 
Alianza Nacional de Pequeños Comerciantes (2020). Mas de 150 mil pequeños negocios han cerrado en México por la pandemia. Recuperado de: https://www.anpec.com.mx/mas-de-150-mil-pequenosnegocios-han-cerrado-en-mexico-por-la-pandemia/.

Arenal, A., Armuña, C., Ramos, S. y Feijoo, C. (2018). Ecosistemas emprendedores y startups, el nuevo protagonismo de las pequeñas organizaciones. Economía industrial, 407, 85-94. Recuperado de: https:/www.mincotur.gob.es/Publicaciones/Publicacionesperiodicas/EconomiaIndustrial/RevistaEconomiaIndustrial/408/ARENAL,\%20ARMU\%C3\%91A,\%20RAMOS\%20Y\%20FEIJOO.pdf.

Ariza, M. (2006). Mercados de trabajo urbanos y desigualdad de género en México a principios del siglo XXI. En De la Garza, E y Salas, C. (Coords.) La situación del trabajo en México. México: Plaza y Valdés.

Cámara de Diputados (2020). Constitución Política de los Estados Unidos Mexicanos. Recuperado de: http://www.diputados.gob.mx/LeyesBiblio/ref/cpeum.htm.

Comisión Económica para América Latina y el Caribe (2020). El desafío social en tiempos de COVID-19. Recuperado de: https://repositorio.cepal.org/bistream/handle/11362/45527/5/S20000325_es.pdf.

Comisión Nacional de los Derechos Humanos (2020). Cuales son los derechos humanos. Recuperado de: https://www.cndh.org.mx/derechos-humanos/cuales-son-los-derechos-humanos.

CONEVAL (2019). Comunicado de Prensa No. 10. Recuperado de: https://www.coneval.org.mx/ SalaPrensa/Comunicadosprensa/Documents/2019/COMUNICADO_10_MEDICION_POBREZA_2008_2018.pdf.

CONEVAL (2020). Informes de Pobreza y Evaluación 2020. Recuperado de: https://www.coneval.org.mx/ coordinacion/entidades/Paginas/Informes_Pobreza_Evaluacion_2020.aspx.

CONEVAL (2020). La política social en el contexto de la pandemia por el virus SARS-Co-V-2 (COVID-19). Recuperado de: https://www.coneval.org.mx/SalaPrensa/Comunicadosprensa/Documents/2020/ Comunicado_06_POLIITICA_SOCIAL_EN_CONTEXTO_COVID_19.pdf.

Cordera, R. y Tello, C. (2010). México la Disputa por la Nación. Perspectivas y Opciones de Desarrollo. México: Siglo Veintiuno.

Cortés, F. (2000). Crisis, miembros del hogar e ingresos. Demos, 13, 35-36.

García, J. y Zapata, E. (2012). El papel de las micro finanzas en la pobreza y desigualdad de las mujeres. Ra Ximhai, 8(1), 99-111. Recuperado de: https://www.redalyc.org/pdf/461/46123324007.pdf.

Gobierno de la República (2013). Plan Nacional de Desarrollo 2013-2018. Recuperado de: file://C:/Users/USER/Downloads/programa_democratizar_productividad.pdf.

Gobierno de Mexico (2020). Jóvenes construyendo el futuro. Recuperado de: https://jovenesconstruyendoelfuturo.stps.gob.mx/.

Gobierno de México (2020). Plan Nacional de Desarrollo 2019-2024. Recuperado de: https://planeandojuntos.gob.mx/.

Gobierno de México (2020). Programa Sectorial de Trabajo y Previsión Social 2020-2024. Recuperado de: https:/www.gob.mx/cms/uploads/attachment/file/561747.

Gómez, C. y Hernández, G. (2016). Competitividad local en el norte de México: El caso de la zona metropolitana de Monterrey. Estudios Regionales en Economía, Población y Desarrollo. Cuadernos de trabajo de la UACJ, 33, 3-34. Recuperado de: https://d1wqtxts1xzle7.cloudfront.net/51823461/ Cuadernos_UACJ_33.pdf?1487263118=\&response-content-disposition=inline\%3B+filename \% 3DCuadernos_UACJ.pdf\&Expires=1623173147\&Signature=GaTW0uMYWeN5SU9uUXQ6- 
im9NC30YtjQwFEZ-HvG19BBbhxraefW0R p dXxZzLvabZsa-7NkVvvxvjcKQlkUv6rfaZVPo depPRUvCtEA6eGYfNiJ3rlW7phZyXiKZOayGNICLQT6cLq2s4cWRa6 g0sTrZZFtjS ZJCQ891 GBEjJ9jO8IMAFNmePoN2hAyE46JCfe eS8zRsX8 oD1K8m29R6WRCv IvzY L37Go9SJ8mR B1Y7IVJHaJ16 8PD9xE-U2UuyGydyr O9yrt3meleSOyOwujzdx3DU5QKh63 PtDxpcSeeZ0qT nup33Dt0CYWKs bfLf5o3cE7zsRRbg_\&Key-Pair-Id=APKAJLOHF5GGSLRBV4ZA.

Impacto Noticias (2020). Estados del norte, los que más aportal PIB y menos reciben. Recuperado de: http:// impactonoticias.com.mx/estatal/estados-del-norte-los-que-mas-aportan-pib-y-menos-reciben/.

Instituto de Investigación para el Desarrollo con Equidad (2020). Impactos del COVID-19 en México. Recuperado de: https://equide.org/wp-content/uploads/2020/09/PP-ENCOVID-19-InfanciaJun-Jul-2020.pdf.

Instituto Mexicano del Seguro Social (2020). Datos abiertos IMSS. Recuperado de: http://datos.imss.gob.mx/. Instituto Nacional de Estadística, Geografía e Informática (2018). Comunicado de prensa núm. 305/18. Recuperado de: https://www.uv.mx/bolsadetrabajo/files/2018/09/iooe2018_07.pdf.

Instituto Nacional de Estadística, Geografía e Informática (2017). Encuesta Nacional sobre la Dinámica de Relaciones en los Hogares. Recuperado de: https://www.inegi.org.mx/contenidos/programas/endireh/2016/doc/endireh2016_presentacion_ejecutiva.pdf.

Instituto Nacional de Estadística, Geografía e Informática (2020). Producto Interno Bruto de México durante el segundo semestre de 2020. Recuperado de: https://www.inegi.org.mx/contenidos/saladeprensa/boletines/2020/pib_pconst/pib_pconst2020_08.pdf.

Instituto Nacional de Estadística, Geografía e Informática (2019). Producto Interno Bruto por Entidad. Recuperado de: https://www.inegi.org.mx/contenidos/saladeprensa/boletines/2019/OtrTemEcon/ PIBEntFed2018.pdf.

Instituto Nacional de Estadística, Geografía e Informática (2020). Cuéntame. Recuperado de: http:// cuentame.inegi.org.mx/economia/default.aspx?tema=E.

Instituto Nacional de Estadística, Geografía e Informática (2020). Encuesta Nacional de Ocupación y Empleo 2019. Recuperado de: https://www.inegi.org.mx/contenidos/programas/enoe/15ymas/doc/ resultados_ciudades_enoe_2019_trim1.pdf.

Kooiman, J. (2010). Governance and governability. En Osborne, S. P. (Ed.) The new public govenrance? Emerging perspectives on the theory and practice of public govenrance. Estados Unidos: Routledge.

Maldonado, B. (2010). Un vínculo necesario: el género y los mercados de trabajo. En Cooper, J. (Ed.) Tiempos de mujeres en el estudio de la economía. México, D. F.: Universidad Nacional Autónoma de México .

Mancini, F. (2016). Cambios y continuidades en la movilidad laboral de la fuerza de trabajo femenina en México. Notas de Población, (102), 229-248. Recuperado de: http://hdl.handle.net/11362/40266.

Martínez, K., Marroquín, J. y Ríos, H. (2019). Precarización laboral y pobreza en México. Análisis económico, 34(86), 113-131. Recuperado de: http://www.scielo.org.mx/scielo.php?pid=S244866552019000200113\&script $=$ sci_arttext.

Martinez, S., Biedma, J. y Ruiz, J. (2015). Las condiciones del entorno emprendedor y el desarrollo económico: un análisis de los países GEM. Revista de Economía Mundial, (41), 181-212. Recuperado de: https://www.redalyc.org/pdf/866/86643415008.pdf.

Martins, H. y Marini, C. (2010). Una guía de gobernanza para resultados en la administración pública. Brasilia: Instituto Publix. 
Mazzucato, M. (2020). Green Growth and the Right Kind of innovation. Recuperado de: https://braveneweurope.com/mariana-mazzucato-green-growth-and-the-right-kind-of-innovation.

Moore, M. (2011). Gestión estratégica y creación de valor en el sector público (4ta ed.). Barcelona: Paidós.

Naciones Unidas (2020). Programa de las Naciones Unidas para el Desarrollo. Recuperado de: https:// www.undp.org/content/undp/es/home/sustainable-development-goals.html.

Oliveira, O. y Ariza, M. (2000). Trabajo femenino en América Latina: un recuento de los principales enfoques analíticos. En Toledo, E. D. (Ed.) Tratado latinoamericano de sociología del trabajo. México: Colegio de México, Flacso, UNAM, FCE.

Secretaría de Relaciones Exteriores (2018). Frontera México-Estados Unidos. Recuperado de: https://mex-eua. sre.gob.mx/images/stories/PDF/actualizacion/b.-Hoja-Informativa-fontera-general-web-2018.pdf.

Secretaría de Relaciones Exteriores (2020). México y Estados Unidos. Recuperado de: https://mex-eua. sre.gob.mx/index.php/frontera.

Secretaría del Trabajo y Previsión Social (2020). Información Laboral, Septiembre 2020. Recuperado de: http://www.stps.gob.mx/gobmx/estadisticas/pdf/perfiles/perfil\%20nacional.pdf.

Sinembargo (2020). La crisis de empleos por COVID-19. Recuperado de: Sinembargo.mx: https://www. sinembargo.mx/05-10-2020/3869320.

Stern, C. (1967). Un análisis regional de México. Demografía y economía, 1(1), 92-117. Recuperado de: https://www.jstor.org/stable/40601735.

Subsistema Nacional de Información Demográfica y Social (2018). Objetivos de Desarrollo Sostenible: Avances y actividades estratégicas. Recuperado de: https://snieg.mx/DocumentaciónPortal/emográfico/sesiones/doc_12018/2_ODS_CESNIDS.pdf.

Tiempo (2020). Estados del norte, los que más aportan PIB y menos reciben. Recuperado de: http://www. tiempo.com.mx/noticia/pib_economia_pactofiscal_aportaciones_participaciones_gobiernofederal_estados_norte_castigados_coronavirus_covid_19/.

Urquidi, V. y Sofía, M. (1975). Importancia económica de la zona fronteriza del norte de México. Foro Internaicional, 16(2), 149-174. Recuperado de: https://www.jstor.org/stable/27754581.

Zalamea, S., y Peña, S. (2015). Articulación de los actores del ecosistema emprendedor como herramienta efectiva para la transición de un proeyecto emprendedor a un proyecto comercialmente viable. Revista de la Facultad de Ciencias Químicas, 11, 64-69. Recuperado de: https://publicaciones. ucuenca.edu.ec/ojs/index.php/quimica/article/view/340.

Zurbiggen, C. (2011). Gobernanza: una mirada desde América Latina. Perfiles latinoamericanos, 19(38), 39-64. Recuperado de: http://www.scielo.org.mx/scielo.php?pid=S018876532011000200002\&script=sci_arttext. 